\title{
Frequency Analysis for The Effect of Pico Tesla-Transcranial Magnetic Stimulation in Epilepsy Patients Using Magnetoencephalography
}

\author{
Photios Anninos ${ }^{1 *}$, Athanasia Kotini', Adam Adamopoulos', Nicolaos Tsagas ${ }^{2}$ \\ 'Labratory of Medical Physics, Department of Medicine, School of Health Sciences, Alexandroupoli, Greece \\ ${ }^{2}$ Department of Electrical Engineering, Polytechnic School, Democritus University of Thrace, Xanthi, Greece
}

Article Info

\section{Article Notes}

Received: August 27, 2018

Accepted: November 05, 2018

\section{*Correspondence:}

Dr. Photios Anninos, Emeritus Professor, Laboratory of Medical Physics, Department of Medicine, School of Health Sciences, Democritus University of Thrace, University Campus, Alexandroupoli 68100, Greece; Telephone No: +302551030392; Fax No: +302551030392;

(C) 2018 Anninos P. This article is distributed under the terms of the Creative Commons Attribution 4.0 International License.

\section{Keywords}

MEG

FFT

PT-TMS

Epilepsy

\section{Abstract}

The purpose of this research is to identify any change in the frequencies of $2-7 \mathrm{~Hz}$ in the brain state of epilepsy patients after pico-Tesla transcranial magnetic stimulation (pT-TMS). It is a noninvasive technique for treating neurological disorders. We used magneto encephalographic (MEG) recordings of 10 epilepsy patients with a whole-head 122 - channel MEG system in a magnetically shielded room of low magnetic noise. The subjects were 5 male and 5 female epilepsy volunteers between 18-42 years of age. Afterwards, external PT-TMS was applied to the above patients. A software program was developed in our lab in order to detect the primary dominant frequency of the power spectra of the MEG obtained from every patient and channel before and after the application of pT-TMS. We found that 7 out of 10 patients $(70 \%)$ had increased their $2-7 \mathrm{~Hz}$ frequencies after the application of pT-TMS. We concluded that frequency analysis is a promising means for the assessment of epilepsy disorders.

\section{Introduction}

Transcranial magnetic stimulation (TMS) is noninvasive and easy to perform method without direct contact with the underlying skin and has been used to investigate a variety of clinical conditions ${ }^{1}$. Its magnetic field strength is about 1.5 to 2 Tesla at the surface of the coil and drops off exponentially with distance from it. A review article $^{2}$ suggested that TMS has provided important insight into the pathophysiological substrate of human epilepsies and it is a valuable tool for diagnostic, prognostic and therapeutic purposes. Magnetoencephalographic (MEG) recordings is a well-established non-invasive method, for investigating human brain activity with whole head neurophysiological measurements. It measures weak magnetic fields generated at the scalp surface by the underlying electrical activity in the brain and it is significant to the diagnosis, classification, and further understanding of epilepsy ${ }^{3}$. To our knowledge, there are only a few reports in the literature investigating epilepsy with MEG and TMS. In a study ${ }^{4}$ with MEG and TMS, suggested that the cortical excitability alteration in focal epilepsy is widely distributed beyond the epileptic focus and the profiles of excitability change correlate with clinical severity in terms of seizure frequency. It was suggested by ${ }^{5}$ that navigated TMS may reveal the functional plasticity and shifts of motor cortical function and epileptic foci may modify cortical inhibition with the navigated TMS results The plan of this study is to discover any modification in the brain state of epilepsy patients with the use of the pT-TMS helmet electronic device, and furthermore that the use of FFT and Transcranial magnetic brain stimulation (TMS) has experimental, diagnostic, and therapeutic perspective. The pico Tesla-TMS electronic tool invented by Anninos and Tsagas $1995^{6}$, is a modified helmet containing up to 122 coils which are arranged in five array groups, so as to cover the 
main 7 brain regions (frontal, vertex, right and left temporal, right and left parietal, and occipital regions) of the subject. It is designed to create pT-TMS range modulations of magnetic flux in the alpha frequency range $(8-13 \mathrm{~Hz})$ of every patient. The pT-TMS device was configured for each individual to produce a square wave (so as to resemble the firing activity of neurons in the brain) ${ }^{7-12}$

\section{Materials and Methods}

In our lab, we used a whole-head 122 channel MEG (Neuromag-122, Neuromag Ltd, Helsinki, Finland) in a magnetically shielded room. The subjects were 5 male and 5 female volunteers between 18-42 years of age.

\section{Data acquisition}

The MEG recordings were filtered with cut off frequencies at $0.3 \mathrm{~Hz}$ and $40 \mathrm{~Hz}$. The MEG sampling frequency was 256 $\mathrm{Hz}$ and the associated Nyquist frequency was $128 \mathrm{~Hz}$, which was well above of the constituent frequency components of interest in our MEG recordings, thus avoiding aliasing artifacts.

The study protocol was approved by the Research Committee of our Democritus University of Thrace. Funding for this work was provided by our collaboration of General Secretariat of Research and Technology, GR and the ERGO AEBE, INC, GR under research program (Grant Number:80623).

Table 1. This table shows the brain regions and the corresponding channels in each brain region.

\begin{tabular}{|l|c|}
\hline Brain Regions & Channels \\
\hline Right Temporal & $1-14,111-120$ \\
\hline Left Temporal & $43-50,55-62,67-74$ \\
\hline Right Parietal & $5-6,11-16,97-100,109,110,115-122$ \\
\hline Left Parietal & $47-52,59-64,71-74,79,80,87-90$ \\
\hline Frontal & $17-42$ \\
\hline Occipital & $75-86,91-96,101-110$ \\
\hline Vertex & $13-16,49-54,61-66,73,74,89,90,99,100,117-122$ \\
\hline
\end{tabular}

\section{Data analysis}

In our lab, we have developed a software program in order to detect the amplitude of the primary dominant frequency of the power spectra of the MEG obtained from every patient and channel after the application of FFT. Then, we looked for the alpha frequency $(8-13 \mathrm{~Hz})$ for calibration of the electronic device and the $(2-7 \mathrm{~Hz})$ frequencies for the analysis to obtain the primary dominant frequencies and the power spectra of the MEG records obtained from each patient and channel after the FFT application ${ }^{13-16}$.

We used the FFT algorithm to obtain the maps of the power spectra of the MEG data. Different colors in the maps represent different dominant frequencies. The numbers in the map squares represent the 122 MEG channels in every part of the brain area according to Table 1.

Every patient was scanned in two sessions. The first session consisted of 2-minute resting state MEG scan and the data were used to establish the subject's alpha frequency $(8-13 \mathrm{~Hz})$ for calibration of the pT-TMS electronic device. In the second session, the pT-TMS electronic device was set to real stimulation. Then, 2 minutes of pre-stimulus baseline MEG data were recorded. Next, 2-minutes of real pT-TMS stimulation were administered with the patient sitting just outside the scanner room. Following these 2-minutes of stimulation, an additional 2-minutes of resting state MEG was acquired to patients.

\section{Results}

Table 1 exhibits the brain regions and the corresponding channels in each brain region. Table 2 , shows the true effect of pT-TMS. In this table, the BS and AS represent the effect prior and after pT-TMS for each of the 10 epilepsy patients in each of the 7 brain regions as we have included in Table 1. Table 3 shows the statistical analysis for the epilepsy patients using unpaired t-test. The results were statistically significant at 7 out of 10 patients (70\%). Table 4 shows the

Table 2. This Table is showing the frequency effect of $\mathrm{pT}$ transcranial magnetic stimulation before the first MEG recording(BS) and after the real stimulation(AS) for each of the 10 epilepsy patients. In this table the first column is the patient number, whereas the other columns the RT is the right temporal brain region, the LT is the left temporal region, the RP is the right parietal region, the LP is the left parietal region, the $\mathrm{F}$ is the Frontal region, the $\mathrm{V}$ is the Vertex and the $\mathrm{O}$ is the Occipital brain region. The last column is shown the figures for the maps of the spatial distribution for the first dominant frequencies before and after pT-TMS for each of the 10 epilepsy patients.

\begin{tabular}{|c|c|c|c|c|c|c|c|c|c|c|c|c|c|c|c|}
\hline $\mathbf{P}$ & $\begin{array}{c}R T \\
B S(H z)\end{array}$ & $\begin{array}{c}\text { RT } \\
\text { AS(Hz) }\end{array}$ & $\begin{array}{c}\text { LT } \\
\text { BS(Hz) }\end{array}$ & $\begin{array}{c}\text { LT } \\
\text { AS(Hz) }\end{array}$ & $\begin{array}{c}\text { RP } \\
\text { BS(Hz) }\end{array}$ & $\begin{array}{c}\text { RP } \\
\text { AS(Hz) }\end{array}$ & $\begin{array}{c}\text { LP } \\
\text { BS(Hz) }\end{array}$ & $\begin{array}{c}\text { LP } \\
\mathrm{AS}(\mathrm{Hz})\end{array}$ & $\begin{array}{c}F \\
B S(H z)\end{array}$ & $\begin{array}{c}F \\
\mathrm{AS}(\mathrm{Hz})\end{array}$ & $\begin{array}{c}0 \\
\mathrm{BS}(\mathrm{Hz}\end{array}$ & $\begin{array}{c}0 \\
\mathrm{AS}(\mathrm{Hz})\end{array}$ & $\begin{array}{c}V \\
B S(H z)\end{array}$ & $\begin{array}{c}V \\
A S(H z)\end{array}$ & Figures \\
\hline 1 & 6 & 5 & 6 & 5 & 4 & 7 & 5 & 7 & 4 & 7 & 5 & 7 & 5 & 7 & Fig3(A,B) \\
\hline 2 & 4 & 4 & 4 & 7 & 4 & 4 & 4 & 7 & 4 & 4 & 5 & 5 & 4 & 7 & Fif4(A,B) \\
\hline 3 & 7 & 7 & 4 & 7 & 6 & 7 & 5 & 7 & 3 & 4 & 7 & 7 & 6 & 7 & Fif5(A,B) \\
\hline 4 & 5 & 7 & 7 & 7 & 4 & 7 & 6 & 7 & 5 & 7 & 4 & 7 & 6 & 7 & Fif6 $(A, B)$ \\
\hline 5 & 6 & 7 & 3 & 7 & 6 & 7 & 4 & 7 & 3 & 7 & 4 & 7 & 3 & 7 & Fif7(A,B) \\
\hline 6 & 7 & 4 & 6 & 5 & 7 & 6 & 6 & 3 & 3 & 3 & 7 & 7 & 7 & 4 & Fif8(A,B) \\
\hline 7 & 6 & 7 & 2 & 7 & 6 & 7 & 3 & 7 & 6 & 7 & 3 & 7 & 5 & 7 & Fif9(A,B) \\
\hline 8 & 6 & 3 & 5 & 7 & 6 & 7 & 4 & 7 & 7 & 5 & 4 & 7 & 3 & 7 & Fif10 $(A, B)$ \\
\hline 9 & 5 & 7 & 5 & 7 & 5 & 7 & 5 & 7 & 5 & 6 & 7 & 7 & 5 & 7 & Fif11 $(A, B)$ \\
\hline 10 & 7 & 7 & 4 & 7 & 7 & 7 & 6 & 7 & 4 & 7 & 7 & 7 & 6 & 7 & Fif12 $(A, B)$ \\
\hline
\end{tabular}


Table 3. This table shows the statistical analysis of 10 epilepsy patients. The results are statistical significant at the level of 0.05 (marked bold)

\begin{tabular}{|c|c|c|c|}
\hline Patients & Mean $\mathbf{f}(\mathbf{B S} \pm \mathbf{S D})$ & Mean $\mathbf{f}(\mathbf{A S} \pm \mathbf{S D})$ & t-test P-values \\
\hline $\mathbf{1}$ & $5.00 \pm 0.82$ & $6.43 \pm 0.98$ & $\mathbf{0 . 0 1 1 7}$ \\
\hline $\mathbf{2}$ & $4.14 \pm 0.38$ & $5.43 \pm 1.51$ & $\mathbf{0 . 0 4 9 6}$ \\
\hline $\mathbf{3}$ & $5.43 \pm 1.51$ & $6.57 \pm 1.13$ & 0.1356 \\
\hline $\mathbf{4}$ & $5.29 \pm 1.11$ & $7.00 \pm 0.00$ & $\mathbf{0 . 0 0 1 5}$ \\
\hline $\mathbf{5}$ & $4.14 \pm 1.35$ & $7.00 \pm 0.00$ & $\mathbf{0 . 0 0 0 1}$ \\
\hline $\mathbf{6}$ & $6.14 \pm 1.46$ & $4.57 \pm 1.51$ & 0.0716 \\
\hline $\mathbf{7}$ & $4.43 \pm 1.72$ & $7.00 \pm 0.00$ & $\mathbf{0 . 0 0 1 9}$ \\
\hline $\mathbf{8}$ & $5.00 \pm 1.41$ & $6.14 \pm 1.57$ & 0.1785 \\
\hline $\mathbf{9}$ & $5.29 \pm 0.76$ & $6.86 \pm 0.38$ & $\mathbf{0 . 0 0 0 4}$ \\
\hline $\mathbf{1 0}$ & $5.86 \pm 1.34$ & $7.00 \pm 0.00$ & $\mathbf{0 . 0 4 4 2}$ \\
\hline
\end{tabular}

symptoms before and after pT-TMS. We observe that 3 out of 10 patients didn't get improvement (no 3,6,8) according to the statistical analysis of Table 3. Especially 2 out of 5 female patients $(40 \%)$ and 1 out of 5 male patients $(20 \%)$ didn't get improvement. Table 4 shows the symptoms of the 10 epilepsy patients before and after pT-TMS as they were evaluated by interview by clinicians. Figure 1 shows the 122 - Channel MEG System and the pT-TMS electronic device. Figure 2 shows an example of MEG record of 9 sec obtained from an epilepsy patient by which after the application of FFT we get the primary dominant frequency from the $2-7 \mathrm{~Hz}$ which is $2.9 \mathrm{~Hz}$.

Table 4. This Table shows the symptoms of 10 epilepsy patients as were evaluated by interview by clinicians before and after stimulation ( $2^{\text {nd }}$ and and $3^{\text {rd }}$ day in the lab) (F:Female; M:Male)

\section{Patients Sex Symptoms before pT-TMS(BS)}

$\mathrm{He}$ is 39 years-old and experienced epileptic seizures since the age of 18. He received carbamazepine(400-

1 M 600 $\mathrm{mg} /$ day) and diazepam $(15 \mathrm{mmg} /$ day. He was experienced seizures about one per week. His MEG was characterized abnormal.

He is 40 years old man with patit mal seizures since the age of 5 . At the age 16 experienced daily tonic-clonic

M generalized seizures which occurred usually at night,but occasionally in the morning upon awakening. His medication was phenytoin(300mg/day),carbamazepine(1200mg/day.His MEG prior to magnetic treatment was abnormal

He is a 39-year-old male patient suffering from idiopathic epilepsy since the age of 10 . He was experienced

$\mathbf{M}$ revealed a background of alpha rhythm activity which was interrupted by series of theta and delta waves. She is 28-year-old female patient suffering daily from tonic-clonic generalized seizures since the age of 10 .

4 F The seizures occur with frequency about 3/day.The anticonvulsant drug therapy was carbamazepine(600mg/ day) and valproid acid (1500mg/day).An MEG taken prior to magnetic treatment showed diffuse abnormalities with high amplitutes of theta and delta waves.

He is a 42-year-old man with epileptic seizures since the age 20. His patient's mother had history of epileptic seizures. His anticonvulsant drug therapy was carbamazepine (400mg.day) and valproid acid(1500mg/day). interrupted by brief of theta and delta waves.

She is 34-year-old female who experienced seizures since the age 4.She is continued to have seizures usually

6 F at sleep. Her medication was carbamazepine(1200mg/day) and valproid acid(1500mg/day) An MEG taken prior to magnetic treatment showed diffuse abnormalities with high amplitudes of theta and delta activities.

She is 20-year-old female patient suffering daily from tonic-clonic generalized seizures since the age of 10 .

The seizures occur with frequency of about 2/day.The treatment drug therapy

7 F Was carbamazepine(1200mg/day)and valproid acid(1500mg/day).An MEG taken prior to magnetic treatment showed diffuse abnormalities with high amplitudes of theta and delta activities.

She is is 18-year-old female with abnormal birth and she experienced seizures since the age 4.Her prior

To magnetic treatment showed diffuse abnormalities with high amplitudes of theta and delta activities She is a 21-year-old women experienced her first tonic-clonic seizure at the age of 18 months. She was the product of normal pregnancy and and was seizure free until the age of 3 years,and the tonic-clonic seizures recurred and was placed on phenytoin.The regimen controlled her seizures until the age of 8 years diazepam(10mg/day)and when she failed to respond she subsequently received sodium valproate(1500mg/ day) and carbamazepine(600mg/day). An MEG taken prior to magnetic treatment showed diffuse abnormalities with high amplitudes of theta and delta activities with seizures up to 10/night.

$\mathrm{He}$ is a 35 -year-old building construction worker with absence(petit mal) seizures since the age 10 . His taken prior to magnetic treatment showed diffuse abnormalities with high amplitudes of theta and delta activities.

Symptoms after pT-TMS(AS)

His seizures were stopped and the MEG was normal

After pT-TMS his MEG

Was Normal with no seizures

After the pt-TMS was characterized with a reduction in the abnormal power spectrum and less seizures

After pT-TMS the MEG has a

reduction in the abnormal power spectrum and without daily seizures

After pT-TMS his MEG was characterized with a reduction in the abnormal power spectrum and without daily seizures

After pT-TMS her MEG is shown a reduction of the abnormal emitted power spectrum

And less sezures.

After pT-TMS her MEG taken

after was characterized with a reduction in the abnormal power spectrum,appearance of alpha rhythm and without daily seizures. After pT-TMS her MEG taken is shown a reduction of the abnormal emitted power spectrum with less seizures

After pT-TMS her MEG taken was shown a reduction of the abnormal emitted power spectrum and with less seizures.

After pT-TMS his MEG taken was shown a good reduction of the abnormal emitted power spectrum and also less seizures. 
The Figures 3-12 represent the maps of the application of FFT on MEG data before and after pT-TMS for each patient. The numbers in each square in the maps give the 122 channels of the MEG system according to Table 1. Different colors represent different primary dominant frequencies $(\mathrm{red}=2 \mathrm{~Hz}$, orange $=3 \mathrm{~Hz}$, yellow $=4 \mathrm{~Hz}$, green $=$ $5 \mathrm{~Hz}$, blue $\geq 6 \mathrm{~Hz}$ ).

The mechanisms by which the application of the pT-TMS attenuated the epilepsy patient's syndrome are unknown.
Nevertheless, one potential reason is that these magnetic fields have been shown to influence the action of the pineal gland which regulates the endogenous opioid functions ${ }^{13}$ and the dopaminergic modulator, GABA ${ }^{14,15}$. Furthermore, on the cellular level, magnetic fields have been shown to influence the properties and constancy of biological membranes and their transport characteristics including the intra and extracellular distributions and fluctuation of calcium ions ${ }^{16}$.

A

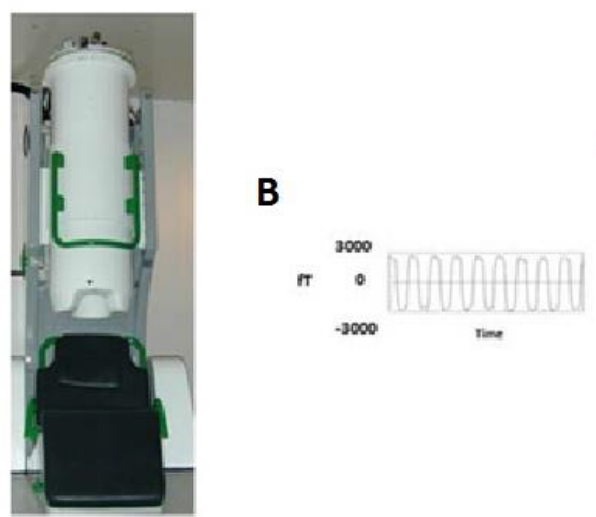

C

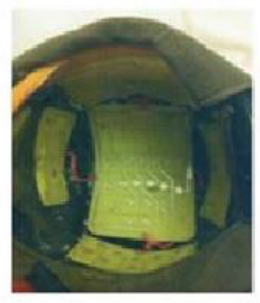

Figure 1: A) The 122 channel MEG system B)The frequency output from the electronic device and C) The coils within the helmet of the Electronic Device
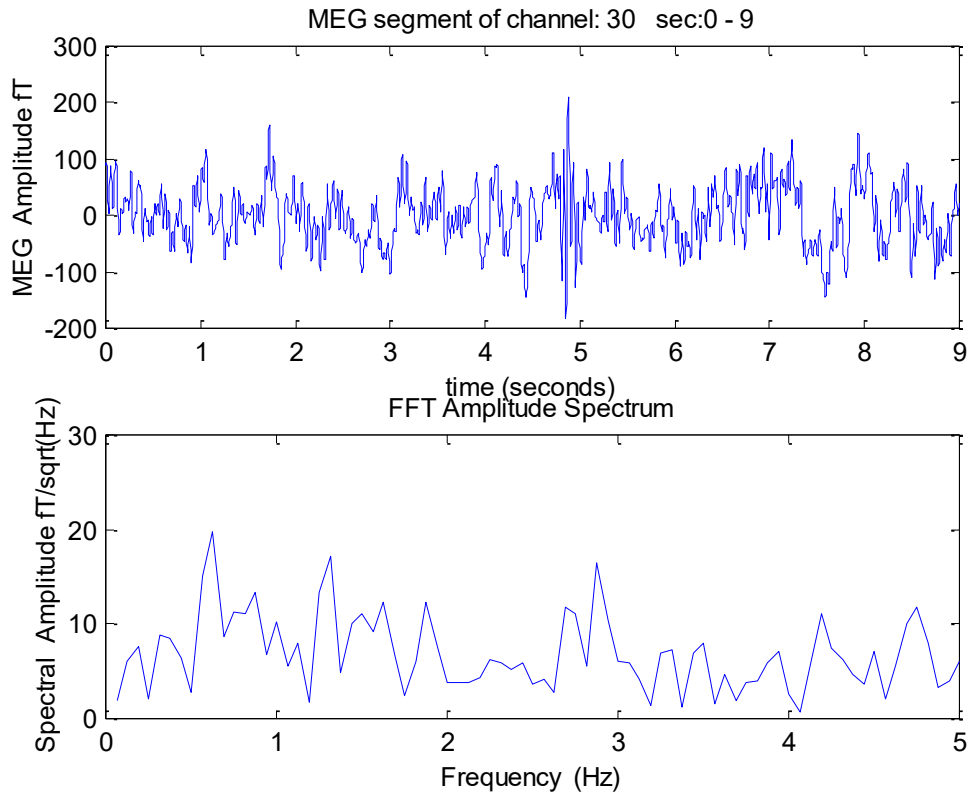

Figure 2: A) A MEG record of 9 sec obtained from an epilepsy patient B) The application of FFT on the MEG record gives that the primary dominant frequency from $2-7 \mathrm{~Hz}$ is $2.9 \mathrm{~Hz}$ 
A

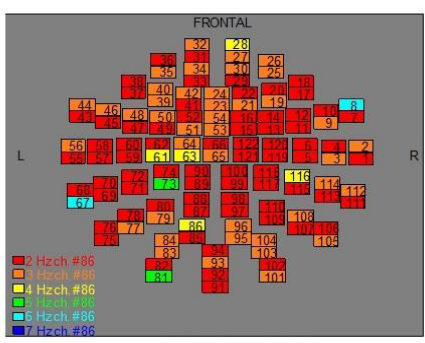

B

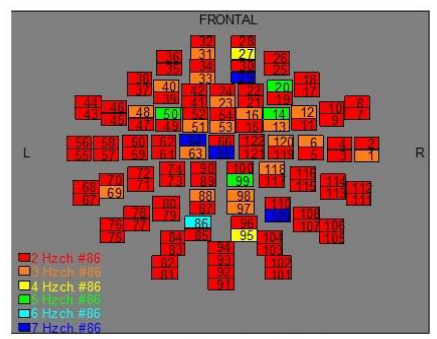

Patient 1

Figure 3: The map of the power spectra of patient $1 \mathrm{~A}$ ) before pT-TMS and B) after pT-TMS

A

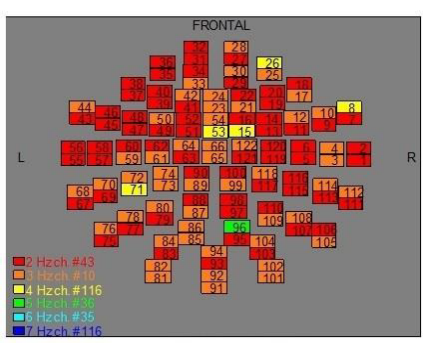

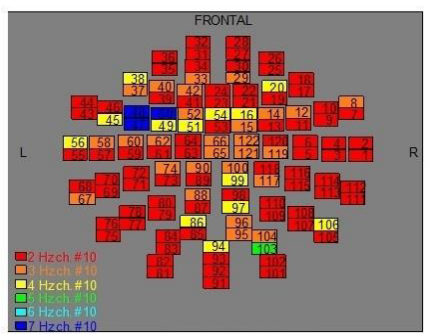

Patient 2

Figure 4: The map of the power spectra of patient $2 \mathrm{~A}$ ) before pT-TMS and B) after pT-TMS

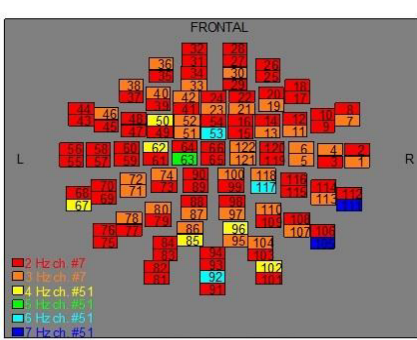

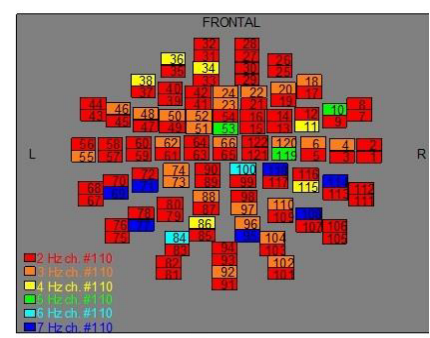

Patient 3

Figure 5: The map of the power spectra of patient $3 \mathrm{~A}$ ) before pT-TMS and B) after pT-TMS

A

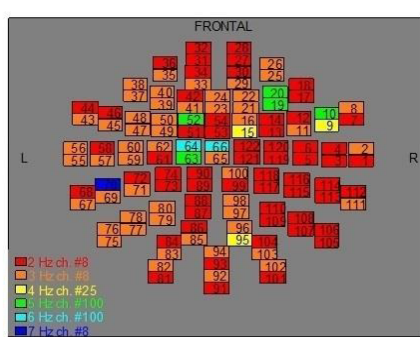

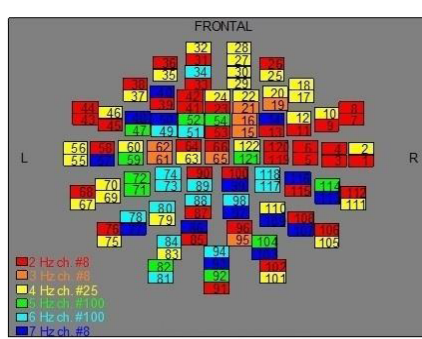

Patient 4

Figure 6: The map of the power spectra of patient 4 A) before pT-TMS and B) after pT-TMS 
A

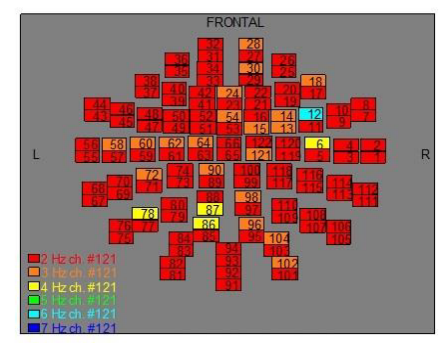

B

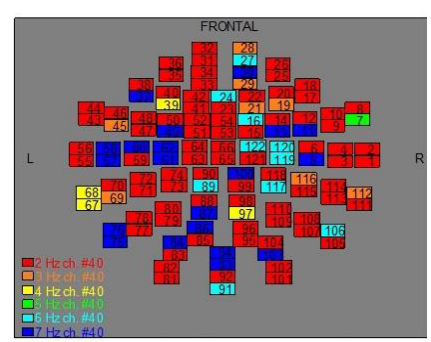

Patient 5

Figure 7: The map of the power spectra of patient 5 A) before pT-TMS and B) after pT-TMS

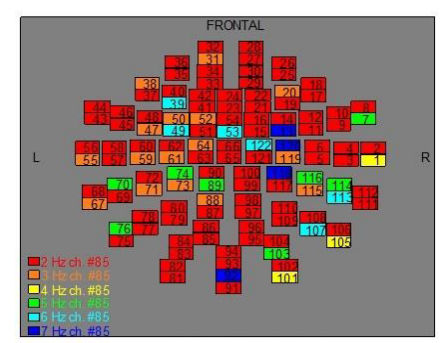

B

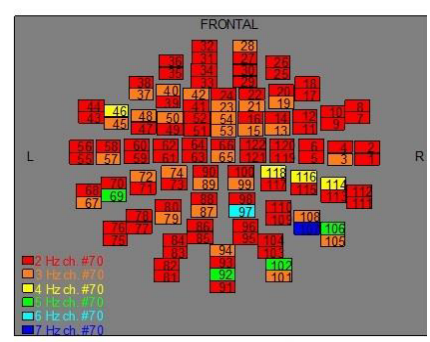

Patient 6

Figure 8: The map of the power spectra of patient 6 A) before pT-TMS and B) after pT-TMS
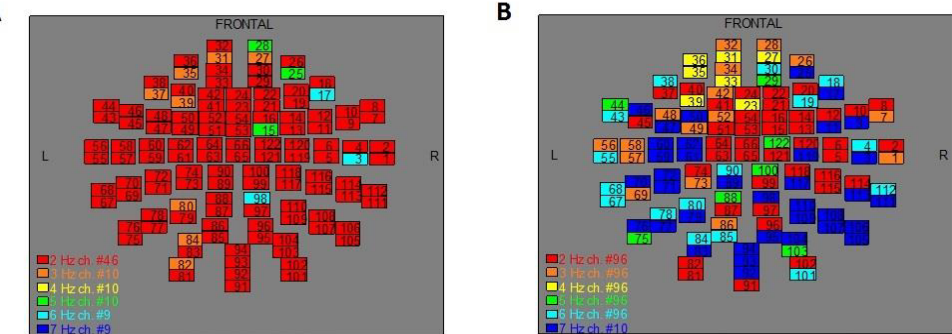

Patient 7

Figure 9: The map of the power spectra of patient 7 A) before pT-TMS and B) after pT-TMS

A

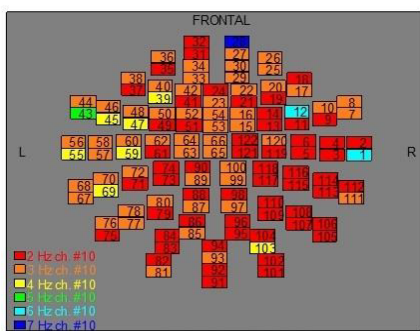

B

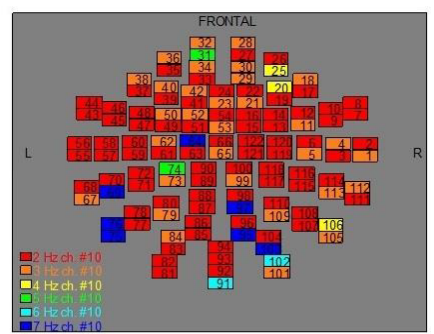

Patient 8

Figure 10: The map of the power spectra of patient $8 \mathrm{~A}$ ) before pT-TMS and B) after pT-TMS 
A

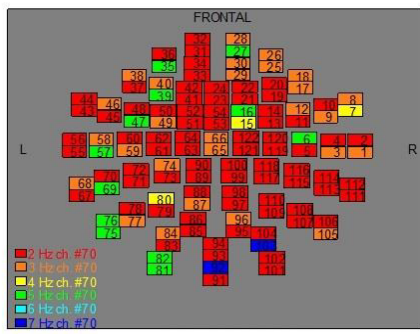

B

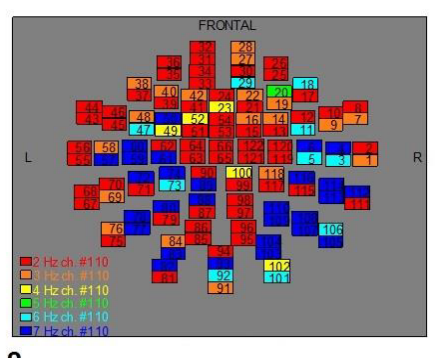

Patient 9

Figure 11: The map of the power spectra of patient 9 A) before pT-TMS and B) after pT-TMS

A

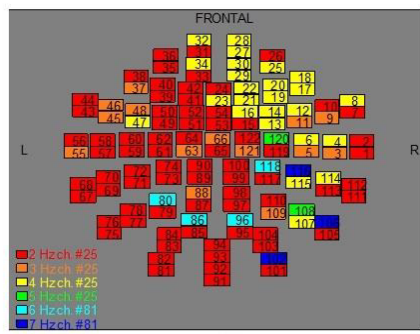

B

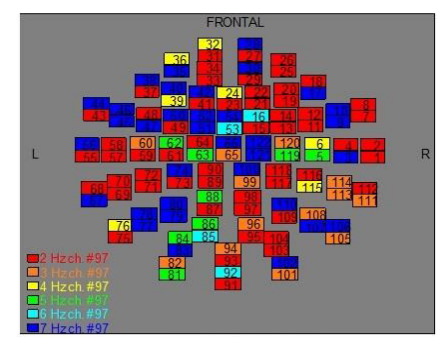

Patient 10

Figure 12: The map of the power spectra of patient $10 \mathrm{~A}$ ) before pT-TMS and B) after PT-TMS

\section{Conclusion}

Therefore, it is possible to conclude that this technique of pT-TMS has some perspective to be a significant noninvasive secure modality in managing epilepsy patients. Nevertheless, additional investigations with more epilepsy patients are needed before firm conclusions can be drawn.

\section{References}

1. Anninos P, Adamopoulos A, Kotini A. MEG as a Medical Diagnostic Tool in the Greek Population. Acta Medica. 2015; 58: 71-78.

2. Anninos P, Kotini A, Anninou N, et al. Meg recordings of patients with CNS disorders before and after external magnetic stimulation. J Integr Neurosci. 2008; 7: 17-27.

3. Anninos P, Adamopoulos A, Kotini A, et al. MEG evaluation of Parkinson's diseased patients after external magnetic stimulation. Acta Neurol Belg. 2007; 107: 5-10.

4. Anninos P, Kotini A, Tamiolakis D, et al. Transcranial magnetic stimulation: A case report and review of the literature. Acta Neurol Belg. 2006; 106: 26-30.

5. Anninos P, Kotini A, Adamopoulos A, et al. Magnetic stimulation can modulate seizures in epileptic patients. Brain Topogr. 2003; 16: 57-64.

6. Anninos PA, Tsagas N. Electronic apparatus for treating epileptic epileptic individuals. USA patent 5453072, 1995.

7. Anninos P, Adamopoulos A, Kotini A. MEG as a Medical Diagnostic Tool in the Greek Population. Acta Medica (Hradec Kralove). 2015; 58(3): 71-8.
8. Anninos P, Kotini A, Anninou N, et al. Meg recordings of patients with CNS disorders before and after external magnetic stimulation. J Integr Neurosci. 2008 Mar; 7(1): 17-27.

9. Anninos P, Kotini A, Adamopoulos A, et al. Magnelic stimulation can modulate seizures in epileptic patients. Brain Topogr. 2003 Fall; 16(1): 57-64.

10. Anninos PA, Tsagas N, Jacobson JI, et al. The biological effects of magnetic stimulation in epileptic patients. Panminerva Med. 1999 Sep; 41(3): 207-15.

11. Kotini A, Anninos P. Alpha, delta and theta rhythms in a neural net model. Comparison with MEG data.J Theor Biol. 2016 Jan 7; 388: 11-4.

12. Kotini A, Mavraki E, Anninos P, et al. Meg evaluation of epileptic activity in the time and frequency domain. J Integr Neurosci. 2008 Dec; 7(4): 463-80.

13. Lissoni P, Esposti D, Esposti G, et al. A clinical study on the relationship between the pineal gland and the opioid system. Neural Transm. 1986; 65: 63-73.

14. Brandbury AJ, Kelly ME, Smith JA. "Melatonin action in the midbrain can regulate dopamine function both behabiourally and biochemically".In Brown GM, Wainwright SD,(Eds). The pineal gland, endocrine aspects,Oxford: Pergamom Press. 1985; 327-332.

15. Nitsche MA, Lampe C, Antal A, et al. Dopaminergic modulation of long-lasting direct current-induced cortical excitability changes in the human motor cortex. Eur J Neurosci. 2006; 23: 1651-7.

16. Ossenkopp KP, Cain DP. Inhibitory effects of acute exposure to low intensity $60 \mathrm{~Hz}$ magnetic fields on electrically kindled seizures in rats. Brain Res. 1988; 442: 255-260. 